\title{
Family Physicians are Complex Care Physicians and Quality of Care Advancement Experts
}

\author{
Marjorie A. Bowman, MD, MPA, and Anne Victoria Neale, PhD, MPH
}

This issue's policy brief provides a good summary point for this issue: family physicians are complex care physicians (in addition to their other many roles). Another article demonstrates that family physicians provide more care during a preventive gynecologic visit than obstetrician-gynecologists. Completing the American Board of Family Medicine Maintenance of Certification modules are associated with higher quality of care. A learning collaborative shows the fruits of their effort to implement patientcentered medical homes. A new risk calculator for colorectal cancer is presented. Pregnant patients are more likely to accept the flu vaccine when presented by a physician rather than a nurse or medical assistant. We can "maladapt" to new information, such as not using aspirin for primary cardiac disease prevention or the low risk of lactic acidosis with metformin. (J Am Board Fam Med 2014;27:1-3.)

Sharma and coauthors ${ }^{1}$ from the Graham Center present this issue's policy brief and suggest that family physicians should be considered "complex care physicians." The evidence? The majority of patients seeking care for 11 of 14 high-cost chronic conditions are more likely to see a primary care physician rather than the corresponding specialist physician, a fact that often is underappreciated. As we know, caring only for conditions requiring chronic care can be more than a full-time job; this article once again suggests that we do not have enough primary care physicians. At minimum, urgent, prevention, behavior change counseling, and health care coordination needs also must be met by this same workforce.

In keeping with this theme of care across the spectrum, Cohen et $\mathrm{al}^{2}$ show that family physicians are about 2.5 times more likely than obstetriciangynecologists to deal with additional nongynecologic health problems at the time of a preventive gynecologic visit. (Editors' note: but they are likely paid the same, which is unfortunate and inappropriate!) This suggests seeing an obstetrician-gynecologist for preventive gynecologic visits also would increase costs because the patient must then see another provider for the additional problems.

Conflict of interest: The authors are editors of the JABFM.

\section{Family Physicians as Quality of Care Advancement Experts}

Family physicians constantly improve the care of their patients. The American Board of Family Medicine has long been a champion of quality of care through both evaluation of physicians' knowledge base and increasingly through efforts to encourage improved care. Manning et $\mathrm{al}^{3}$ provide information on the measurable impact of American Board of Family Medicine Maintenance of Certification modules on the quality of care family physicians deliver. The bottom line reinforces why this form of continuing education is so important, albeit underappreciated: both process measures and intermediate outcome measures for type 2 diabetes care improved for those physicians completing the modules specific to the measures compared with those who did not. Another important finding is that all groups, whether participating or not participating in the modules, showed improvements over time.

Continuing medical education is also an integral aspect of academic practice: teaching about a subject or responding to learner questions can help academic physicians keep up to date on recent recommendations. Callegari et $\mathrm{al}^{4}$ find this to be the case with changes in recommendations for levonorgestrel intrauterine devices; physicians teaching residents or fellows were more likely to follow the recently more inclusive indication guidelines. 
To improve the nation's health, family physicians also have been highly involved in practicebased research, which has moved beyond descriptive and cross-sectional studies and is now important for longitudinal pragmatic studies that require long-term evaluation and practice engagement. Yawn et $\mathrm{al}^{5}$ draw on years of experience to distill 8 general strategies to "prevent voltage drop" (ie, loss of clinic engagement/momentum or even study drop out). These strategies run the spectrum for enrollment and data collection and entry as well as continued use of the implemented practice change.

One type of practice-based research has centered on improving family medicine practice management and delivery of care through various efforts, including learning collaboratives that make intense efforts to change practice. The data presented by Halladay et $\mathrm{al}^{6}$ confirm the importance of registry implementation and protocol use as key elements of improving patient care for patients with diabetes in the 42 studied practices.

\section{Research by Family Physicians for Family Physicians Can Improve Future Care}

Wells et $\mathrm{al}^{7}$ use a large database to create an algorithm to determine the need for colorectal cancer screening that goes beyond the typical simplistic formulae of today (such as "first colorectal screening at age 50"). The statistical techniques used in this article are impressive. The authors created a free online risk calculator using data from the Multiethnic Cohort study, which followed $>180,000$ patients for the development of colorectal cancer for up to 11.5 years through linkage with cancer registries. The final 12 variables used for the algorithm are readily available in medical records or by simple patient queries. It is notable that this risk calculator is not for people with a history of polyps. Use of this risk calculator could increase the accuracy of screening, sometimes preventing unnecessary screenings and sometimes prompting the discovery of cancers at a treatable stage.

With an observational study, Zakrzewski et $\mathrm{al}^{8}$ observed that the flu vaccine was less likely to be given to pregnant patients when initiated through a medical assistant- or nurse-driven protocol than a physician-based protocol, even though the vaccine was more frequently offered by nurses or medical assistants. Simply offering the vaccine is insuffi- cient. Given common patient reluctance about the flu vaccine, the low uptake may be improved with a more complete and trusted discussion of vaccine pros and cons.

In a study of lifestyle change, Mitchell et al ${ }^{9}$ report that providing biomarker feedback to patients (including typical vital signs as well as 2 relatively unknown markers called homeostatic insulin resistance and nuclear magnetic resonance lipoprotein profile) was not associated with substantial improvements in outcome.

Do patients "hear" us, particularly in discussions about sensitive subjects such as weight? We are thankful that, in the Project CHAT study, Bodner et $\mathrm{al}^{10}$ found that audio recordings and self-reports of discussions during visits verify that patients do hear the physician discuss weight.

National guidelines can help, and they can hurt. It can take time, often years, to get health care providers to start following newly developed evidence-based guidelines, and, as noted by Hissett et al, ${ }^{11}$ it can also take years to stop following national advice that is later proven incorrect, as is the case for aspirin for the primary prevention of heart disease (although it is still recommended for secondary prevention of recurrent heart disease). In the meantime, physicians may be graded and paid incentives based on adherence to old, faulty recommendations. Up-to-date physicians who modify their prescribing practices based on the newer data may find this particularly galling. Perhaps when the US Preventive Services Task Force revises its current recommendations, which is underway, then practice will change back to the avoidance of aspirin for primary prevention.

The article aptly titled "MALAdaptive: Do We Avoid Metformin Unnecessarily?" also highlights the time it takes to change practice after new information is introduced. Metformin-associated lactic acidosis (MALA) has discouraged physicians from prescribing metformin for years, yet the incidence of MALA is seriously overestimated (current estimates are 3-4.3 cases per 100,000 patient-years of exposure), and metformin is otherwise an excellent drug for diabetes. As Terpening ${ }^{12}$ notes, most lactic acidosis in patients taking metformin is probably metformin coincident lactic acidosis rather than metformin associated lactic acidosis. Time for change! Patient has mild renal impairment? Metformin is okay. Patient has some congestive heart failure? Met- 
formin is okay. Read the article for more specific information.

Several other articles round out this $7 A B F M$ issue. Women employed after childbirth are less depressed. Gjerdingen et $\mathrm{al}^{13}$ identified lower depressive symptoms in postpartum women who are employed and in those with higher social support (other than partner support) after controlling for multiple risk factors. Hooker et $\mathrm{al}^{14}$ found that sterile pyuria is common in patients hospitalized for a variety of infections. The causes and significance of sterile pyuria are unclear, yet it can confuse patient diagnosis and treatment. In addition, despite higher risks of fractures, Dreyfus et $\mathrm{al}^{15}$ noted that bone mineral density testing is underused in adults with intellectual disability, especially those men and women with Down syndrome.

\section{Pisacano Scholars}

Each year the ABFM selects a small cadre of upand-coming family medicine trainees to join the illustrious society of Pisacano Scholars. The latest group of future leaders is welcomed in this issue. ${ }^{16}$ We also include a special communication from previous Pisacano Scholars reflecting on the future of family medicine. ${ }^{17}$

\section{References}

1. Sharma MA, Cheng N, Moore M, Coffman M, Bazemore AW. Patients with high-cost chronic conditions rely heavily on primary care physicians. J Am Board Fam Med 2014;27:11-2.

2. Cohen D, Coco A. Do physicians address other medical problems during preventive gynecologic visits? J Am Board Fam Med 2014;27:13-8.

3. Galliher JM, Manning BK, Petterson SM, et al. Do professional development programs for maintenance of certification (MOC) affect quality of patient care? J Am Board Fam Med 2014;27:19-25.

4. Callegari LS, Darney BG, Godfrey EM, Sementi O, Dunsmoor-Su R, Prager SW. Evidence-based selection of candidates for the levonorgestrel intrauterine device (IUD). J Am Board Fam Med 2014;27:26-33.

5. Yawn BP, Dietrich A, Graham D, et al. Preventing the voltage drop: keeping practice-based research network (PBRN) practices engaged in studies. J Am Board Fam Med 2014;27:123-35.
6. Halladay JR, DeWalt DA, Wise A, et al. More extensive implementation of the Chronic Care Model is associated with better lipid control in diabetes. J Am Board Fam Med 2014;27:34-41.

7. Wells BJ, Kattan MW, Cooper GS, Jackson L, Koroukian S. ColoRectal Cancer Predicted Risk Online (CRC-PRO) calculator using data from the Multi-Ethnic Cohort Study. J Am Board Fam Med 2014;27:42-55.

8. Zakrzewski L, Sur DK, Agrawal N. Staff versus physician vaccine protocols for influenza immunization during pregnancy. J Am Board Fam Med 2014;27: $56-60$.

9. Mitchell NS, Manning BK, Staton EW, Emsermann CD, Dickinson LM, Pace WD. Outcomes of biomarker feedback on physical activity, eating habits, and emotional health: from the Americans in Motion-Healthy Intervention (AIM-HI) study. J Am Board Fam Med 2014;27:61-9.

10. Bodner ME, Dolor RJ, Østbye T, et al. Accuracy and congruence of patient and physician weight-related discussions: from Project CHAT (Communicating Health: Analyzing Talk). J Am Board Fam Med 2014;27:70-7.

11. Hissett J, Folks B, Coombs L, LeBlanc W, Pace WD. Effects of changing guidelines on prescribing aspirin for primary prevention of cardiovascular events. J Am Board Fam Med 2014;27:78-86.

12. Terpening C. MALAdaptive: do we avoid metformin unnecessarily? J Am Board Fam Med 2014; 27:136-41.

13. Gjerdingen D, McGovern P, Attanasio L, Johnson PJ, Backes Kozhimannil K. Maternal depressive symptoms, employment, and social support. J Am Board Fam Med 2014;27:87-96.

14. Hooker JB, Mold JW, Kumar S. Sterile pyuria in patients admitted to the hospital with infections outside of the urinary tract. J Am Board Fam Med 2014;27:97-103.

15. Dreyfus D, Lauer E, Wilkinson J. Characteristics associated with bone mineral density screening in adults with intellectual disabilities. J Am Board Fam Med 2014;27:104-14.

16. Ireland J. Pisacano Leadership Foundation names 2013 Pisacano scholars. J Am Board Fam Med 2014; 27:162-6.

17. Doohan NC, Duane M, Harrison B, Lesko S, DeVoe JE. The Future of Family Medicine version 2.0: reflections from Pisacano scholars. J Am Board Fam Med 2014;27:142-50. 